\title{
Valve-in-valve apps: why and how they were developed and how to use them
}

\author{
Vinayak Bapat*, FRCS, CTh \\ Department of Cardiothoracic Surgery, Guy's and St. Thomas' Hospital, London, United Kingdom
}

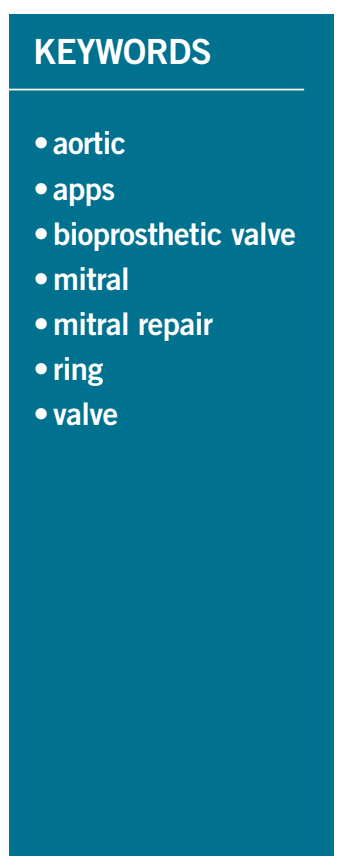

\begin{abstract}
Rapid growth in transcatheter heart valve (THV) technology has led to its use in the treatment of degenerated bioprosthetic surgical heart valves (SHV) and failed mitral repairs. Multiple reports of valve-in-valve (VIV) and valve-in-ring (VIR) procedures have appeared in the literature during the last three years. The success of a VIV procedure is based on the correct identification of the surgical valve/ring, choosing the correct size of the THV and its subsequent accurate placement. There are, however, SHV and mitral rings implanted in the last two decades which differ in design, dimensions and fluoroscopic appearances. In the past, the users had to search through the literature to find out case-specific information. Unlike publications, by using the smartphone platform, we have collated vast amounts of available information, which can take the user through various links to present specific information about a clinical scenario relevant to their patient. Once downloaded, the information is available without the need for an Internet connection and can help in planning and performing a VIV/VIR procedure. The app platform also allows the addition of new material easily in the pre-existing user interface.
\end{abstract}

\footnotetext{
*Corresponding author: Department of Cardiothoracic Surgery, 6th Floor, East Wing, St. Thomas' Hospital, London, SE1 7EH, United Kingdom.E-mail: vnbapat@yahoo.com
} 


\section{Introduction}

The introduction of transcatheter heart valve (THV) technology in 2002 led to transcatheter aortic valve implantation (TAVI) becoming an established modality of treatment in inoperable and highrisk patients with aortic stenosis ${ }^{1,2}$. Since its commercialisation in Europe, the field has grown as a result of clinical need not only in the treatment of aortic stenosis but also for novel applications such as bioprosthetic surgical heart valve (SHV) degeneration, aortic regurgitation and the treatment of native mitral valve disease $\mathrm{e}^{3-16}$.

Valve-in-valve (VIV) and valve-in-ring (VIR) where a THV is placed within a degenerated SHV and a mitral ring, respectively, are now recognised treatments. Two THVs (the SAPIEN XT [Edwards Lifesciences, Irvine, CA, USA] and the CoreValve [Medtronic Inc., Minneapolis, MN, USA]) now have CE mark approval for this indication. The Portico ${ }^{\mathrm{TM}}$ (St. Jude Medical, St. Paul, MN, USA) and JenaValve (JenaValve Technology GmbH, Munich, Germany) have also recently been used ${ }^{17,18}$. Although VIV/VIR may be an overtly easier operation when compared to aortic valve replacement, i.e., implanting a THV within a scaffold of an SHV/ring, design differences between various SHVs and rings can pose certain challenges, such as choosing the correct size of THV and the optimal placement of a THV within the SHV/ring ${ }^{9-15}$. Complications such as malposition, embolisation, coronary obstruction and high residual gradients have been reported after VIV and may reflect limitations of this therapy ${ }^{16}$. Hence, it is of paramount importance to know the details of the SHV/ring being treated, and also to know its compatibility with the THV under consideration so as to achieve the best possible result ${ }^{9,11}$.

With multiple valve and ring types available and each manufactured in multiple sizes, this information is not easily available for physicians. With the popularity of handheld technology booming, smartphones and tablets have become an integral part of daily life. Rapid expansion of software tools has led to the development of a vast variety of apps including those for medical use for patients as well as for physicians. The Apple iTunes Store and Google Play are home to over a million apps each, and between them over one billion downloads. Within these, there are over 100,000 medical apps currently available, and it is estimated that, by 2015 , over 500 million smartphone users worldwide will use medical apps. The app interface allows collation of complex data, especially when it involves thousands of permutations and combinations, and also allows use of data in various formats such as text, images, animation and movie clips, making it a perfect tool to provide guidance on the VIV and VIR procedures. By utilising the smartphone platform we could collate this complex information in the form of apps, so as to provide information specific to a clinical scenario that can be quickly and simply obtained, and which could be of help in planning and performing a VIV or VIR procedure.

In this article we discuss the need for such an application, how it was developed and how to use it for planning a VIV or VIR procedure.

\section{Need for apps}

There are five main areas to be considered to ensure the success of a VIV/VIR procedure. These are:

1. understanding the design of the failing SHV/ring;
2. understanding THV design and confirming its suitability for VIV/ VIR use;

3. choosing the correct size of THV prosthesis for the existing SHV/ring;

4. the ideal implantation position of the THV within the existing SHV/ring;

5. the mode of failure (presence of pannus).

The apps were developed with these features in mind, addressing each aspect in detail and in correct sequence to ensure that operators were provided with all relevant information to ensure a successful procedure. The aim was to build a workflow that could provide information specific to a clinical scenario, with each step taking the user from important generic information about a particular SHV/ ring to very detailed information about a particular combination of SHV and THV, including the end result. Figure 1 shows the workflow, containing information that led to the development of the app.

\section{Key components for planning a VIV/VIR procedure UNDERSTANDING A SURGICAL HEART VALVE DESIGN}

The key element in performing a VIV is the correct identification of the $\mathrm{SHV}^{9}$. As the VIV procedure is performed under fluoroscopy, information about important features such as components and design features, which can impact on fluoroscopic appearance, is essential for planning VIV. SHVs are also manufactured in multiple label sizes

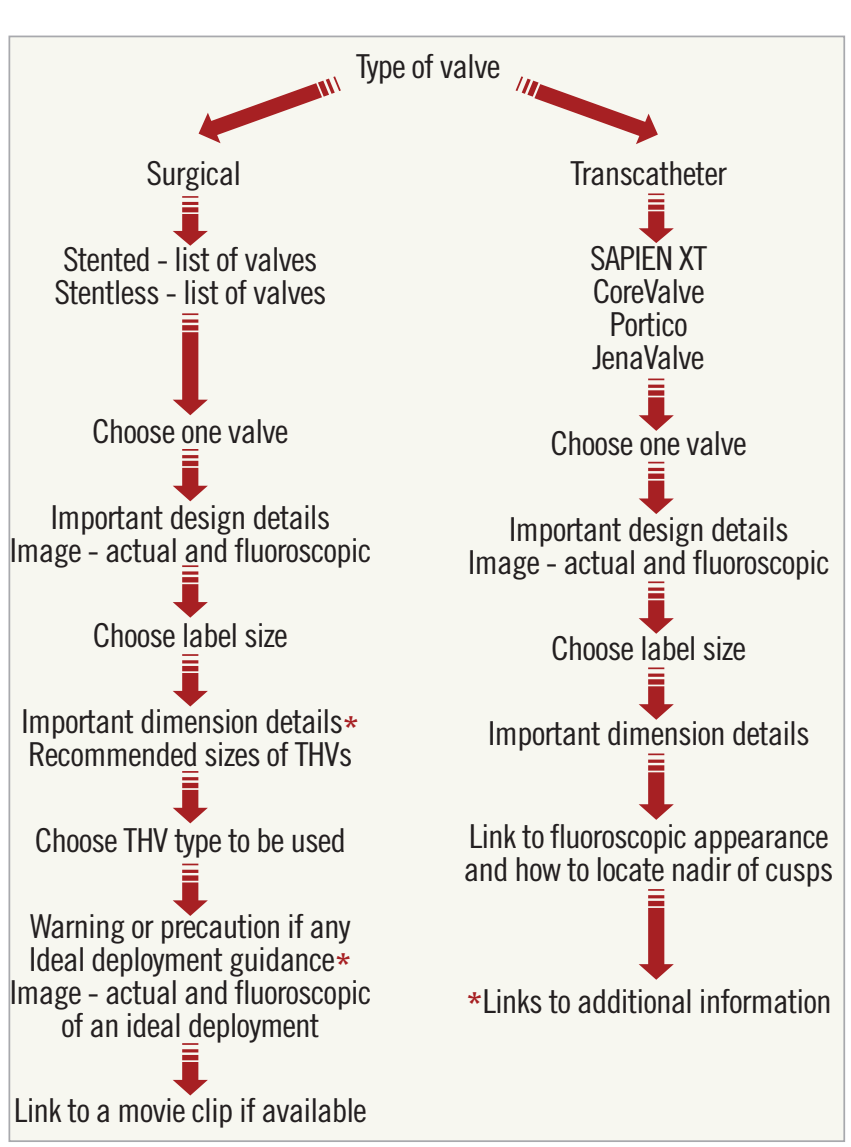

Figure 1. Flow chart for the app. Information needed to build the app arranged as a workflow, which helped acquire the text, images and movie clips required to build the apps. Reproduced with permission from Bryn Mawr Communications LLC 23 
and, although a numbering system is followed, the dimensions of similar label size SHVs from various manufacturers are not the same?

To obtain information on design and dimensions, major heart valve manufacturers were contacted, and all sizes of the commercially available 17 stented and eight stentless aortic valves and seven mitral stented valves were obtained. Important valve-related data obtained were:

1. Stented valves

a. leaflet type

b. position of leaflet relative to stent frame (inside or outside)

c. stent internal diameter (ID) from manufacturer charts

d. stent height

e. true ID measured with Hegar's dilators

2. Stentless SHVs

a. type (root of valve)

b. tissue annulus diameter

c. true ID measured with Hegar's dilators

The design characteristics of each SHV were documented with high-quality photographs and fluoroscopic imaging (Figure 2). Fluoroscopic guidance as well as guidance on dimensions have been published elsewhere'.

\section{TRANSCATHETER HEART VALVE DESIGN}

All sizes of the four THVs in clinical use for VIV (Edwards SAPIEN XT [Edwards Lifesciences], Medtronic CoreValve [Medtronic], St. Jude Portico [St. Jude Medical] and JenaValve [JenaValve Technology]) were obtained from their manufacturers (Figure 3). These were also photographed and their fluoroscopic images were obtained. As VIV is a fluoroscopically directed procedure, it is vital to be familiar with the most important fluoroscopic landmark, i.e., the nadir of the leaflets, which allows correct placement of the THV within the SHV (Figure 4).

Important information about dimensions was obtained. This is relevant to VIV and VIR procedures, as some SHV/rings may be too small or too large for the currently available THV sizes.
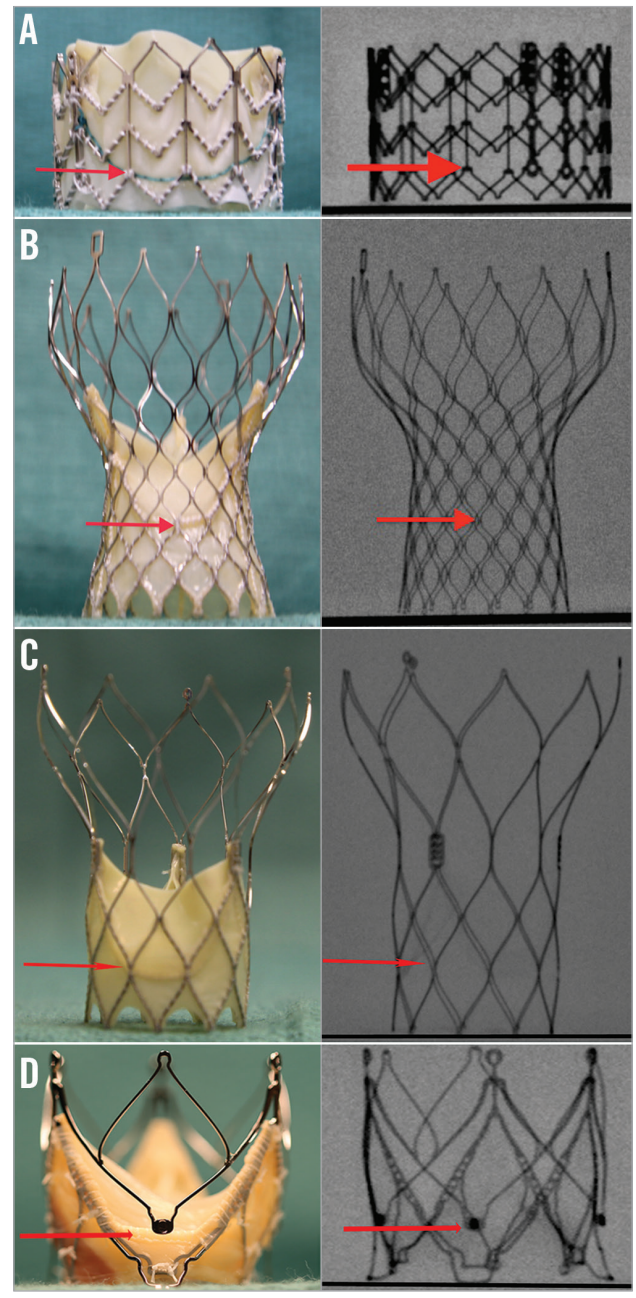

Figure 3. Transcatheter heart valves and level of nadir of leaflets. As $V I V$ and VIR procedures are performed under fluoroscopy, it is important to know the level of nadir of leaflets (red arrow) during the procedure. A) SAPIEN XT. B) CoreValve. C) Portico. D) JenaValve. Reproduced with permission from Bryn Mawr Communications $L L C$ *23

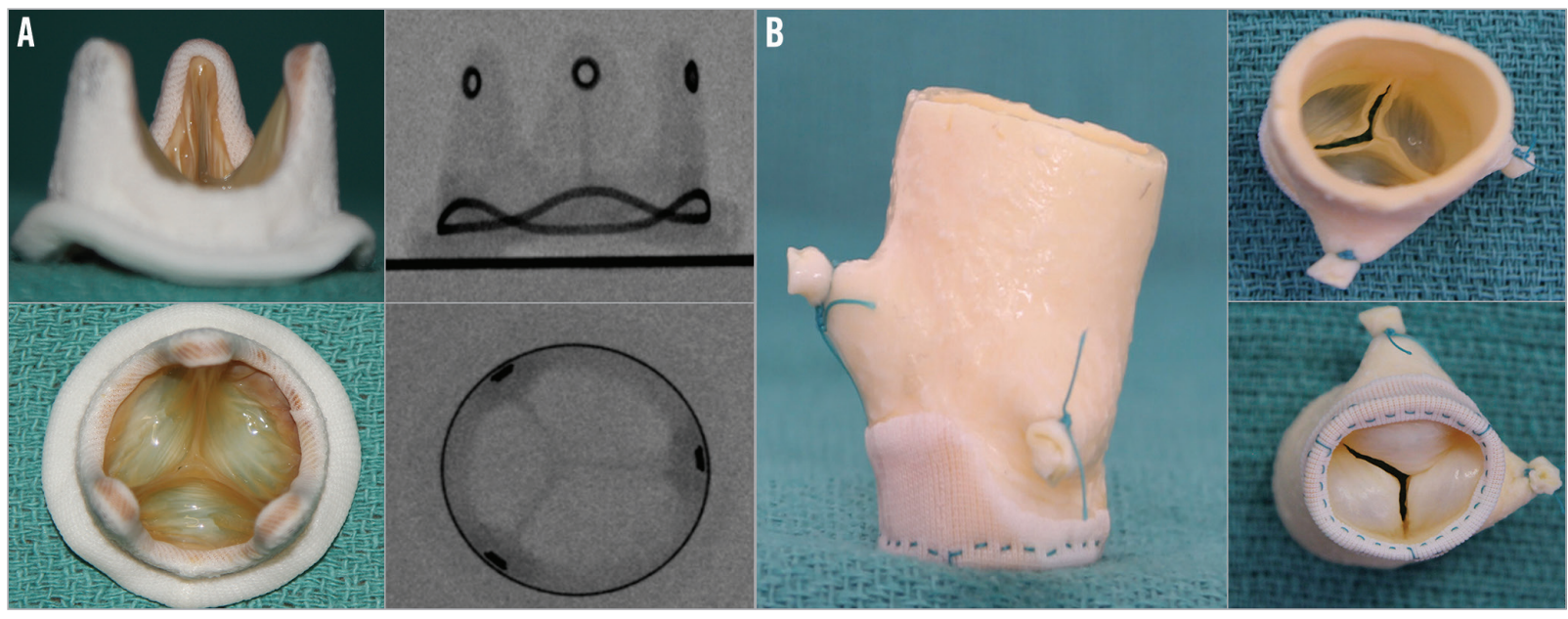

Figure 2. Different types of surgical heart valve. A) Example of a stented SHV - Hancock II. Side and top profile photographs and fluoroscopic images. B) Example of a stentless SHV - Toronto SPV (St. Jude Medical) porcine root. Side, top and inflow photographs. Stentless valves are not visible under fluoroscopy. 


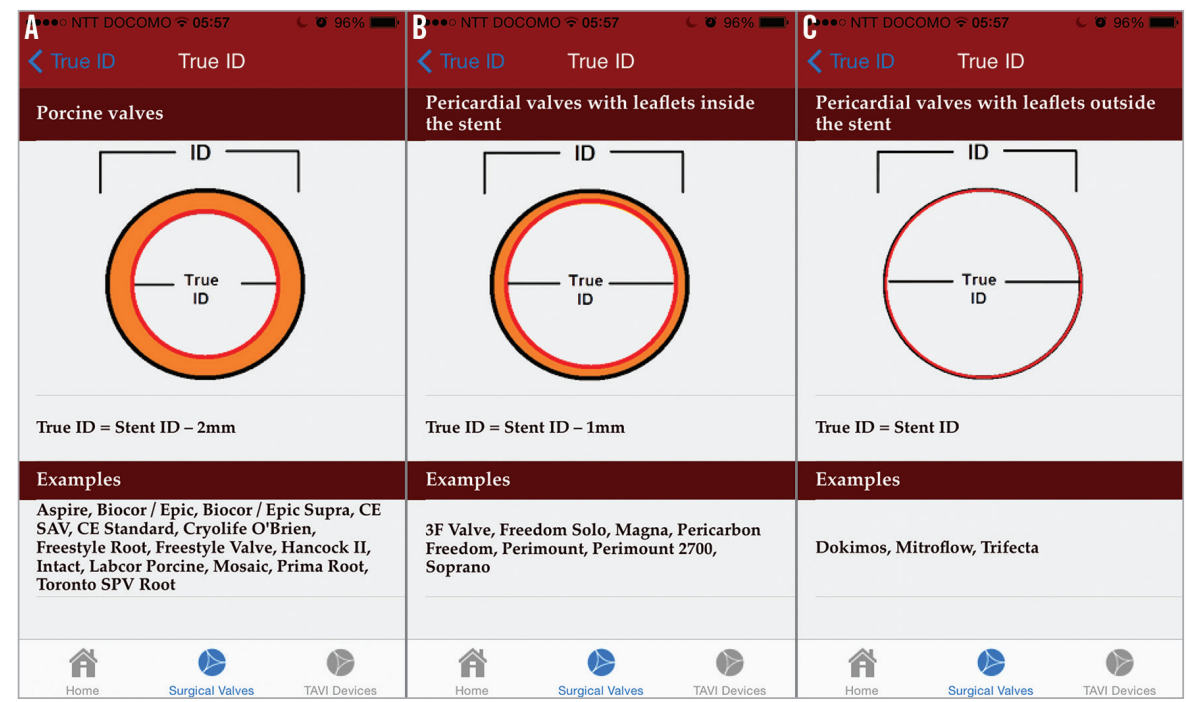

Figure 4. Effect of SHV design on the stent ID. A) Porcine valves. B) Pericardial valves with leaflets sutured inside the stent. C) Pericardial valves with leaflets sutured outside the stent. Reproduced with permission from Bryn Mawr Communications LLC ${ }^{* 23}$

\section{CORRECT SIZING OF THV AND THE CONCEPT OF TRUE ID}

The true ID of an SHV is different from the stent ID $^{10}$. The stent ID is based on the stent diameter of the valve without leaflets and the true ID of an SHV is the inner diameter of the valve, with the presence of the valve leaflets. This diameter varies, as shown in Figure 3, with the type and placement of leaflets.

Essentially, when compared to the stent ID, the true ID is less by at least $2 \mathrm{~mm}$ in all porcine valves, less by $1 \mathrm{~mm}$ in all pericardial valves with leaflets mounted inside the stent, and equal to the true ID when pericardial leaflets are mounted outside the stent ${ }^{10}$. The size of THV should be chosen according to the true ID as it is the most relevant measurement during a VIV procedure. It is important to rule out pannus ingrowth as it may reduce the true ID further. Pannus can be easily identified by non-invasive investigations such as TEE.

Investigations, including multicentre and single-centre experiences with special attention paid to reported complications to ascertain compatibility, were undertaken before THV size recommendation was provided ${ }^{5,10,12,16,19-21}$.

\section{IDEAL IMPLANT POSITION OF THV WITHIN A GIVEN SHV}

To avoid malposition, the THV must be optimally placed within an SHV (Figure 5A, Figure 5C) ${ }^{22}$. Too deep a placement can hamper adequate function of the THV (Figure 5B). Similarly, too high an implantation can result in embolisation (Figure 5D). As the sewing ring of the SHV is its narrowest diameter, it can provide a good anchor for a THV. As the procedure is performed under fluoroscopy, locating the level of the sewing ring under fluoroscopy is essential.

The sewing ring, however, is not radiopaque in all SHVs. In fact, valves may have either a visible sewing ring or a visible stent frame or no radiopaque features at all (Figure 6). In valves with a visible sewing ring, to obtain an ideal implant position is easy irrespective of the supraor intra-annular design of the valves, whereas in valves with a visible stent frame this design feature has to be taken into consideration.

To simplify this for the users, THVs were implanted in an optimal implant position in each type of SHV and were photographed, in addition to the taking of fluoroscopic images. These represent the ideal or optimal end result for every VIV combination (Figure 7). Users can easily refer to these combinations depending on the THV they choose, and can aim to achieve the final implant position similar to the image for an optimal result.

\section{MOVIE CLIP}

Finally, short movie clips showing real-life cases were included to make this app as clinically relevant and useful as possible. It is
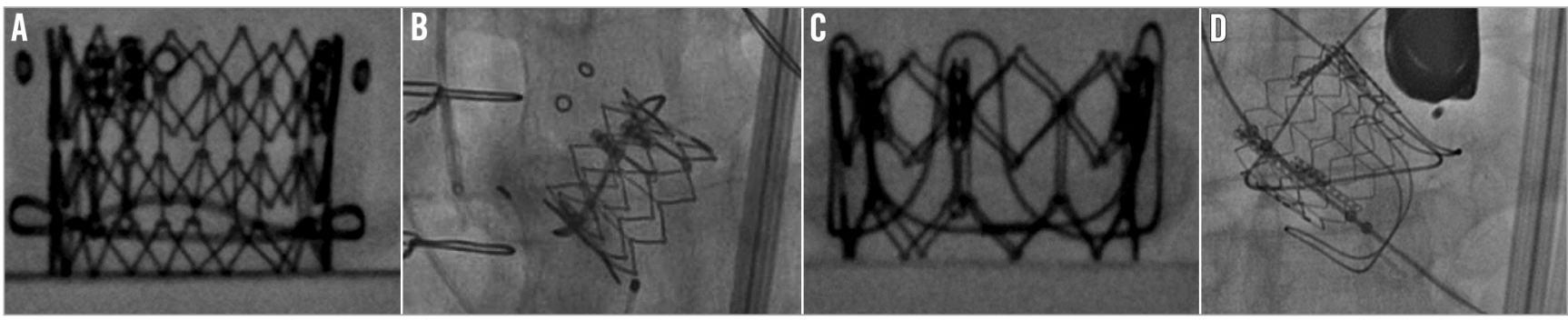

Figure 5. Importance of understanding the level of the sewing ring of the SHV. A) Ideal position for SAPIEN XT in Hancock II SHV. B) SAPIEN XT placed very low into a failed Hancock II SHV. C) Ideal position for SAPIEN XT in CE SAV porcine SHV. D) Displacement of SAPIEN XT after a high placement within a failed CE SAV porcine valve, which led to embolisation. 
especially important when the placement is tricky due to a paucity of fluoroscopic markers and when the user is not familiar with a particular SHV design.

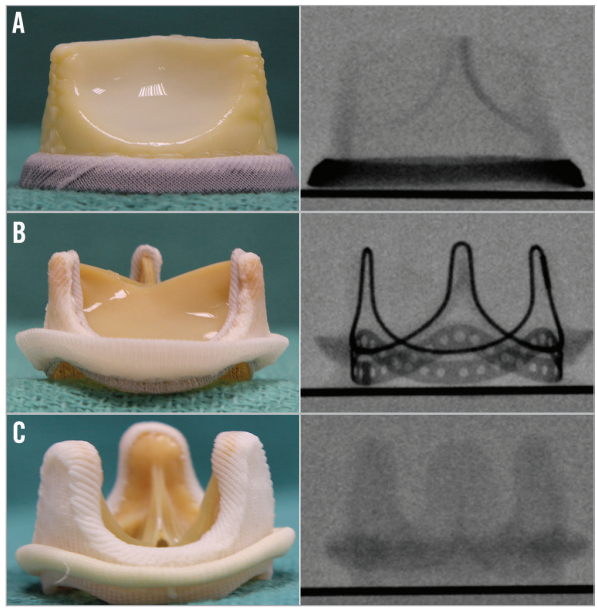

Figure 6. Fluoroscopic appearances for different SHV. A) SHV with sewing ring visible under fluoroscopy. Example demonstrated here is Mitroflow. B) SHV with stent frame visible under fluoroscopy. Example demonstrated here is Perimount. C) SHV with no part visible under fluoroscopy. Example demonstrated here is Aspire ${ }^{\mathrm{TM}}$ (Vascutek, Leeds, UK).

\section{STENTLESS VALVES}

The highest incidence of malposition and coronary obstruction has been reported with stentless valves ${ }^{16}$. Stentless valves can be implanted either as root replacement or in a subcoronary fashion ${ }^{3}$ (Figure 8). The former includes re-implantation of the coronary buttons and hence is at lower risk for coronary obstruction after a VIV procedure. On the other hand, in a subcoronary implantation technique, the suture line is in close proximity to the coronary ostia and there is a higher risk of coronary obstruction after a VIV procedure. Stentless valves lack a stent frame and hence are not visible under fluoroscopy. These two features make VIV in a stentless valve challenging. Tips and tricks used during a VIV implantation within stentless valves have been included in the app as a separate section to help users ensure a successful outcome.

\section{UNKNOWN VALVES}

This section provides in vitro and in vivo fluoroscopic images of all stented valves, which can be matched to the fluoroscopic image of the patient's SHV to identify/confirm the type of SHV. This is important when information about the implanted prosthesis is not known.

\section{MITRAL RINGS}

Rings were also obtained from all manufacturers. The differences were characterised in relation to a VIR procedure with a SAPIEN XT THV:

a. complete or incomplete;

b. rigid, semi-rigid or flexible;

c. dimensions - anteroposterior, lateral (commissure to commissure) and area;

d. fluoroscopic appearance.

A SAPIEN XT of an appropriate size was then implanted in an ideal position and high-quality photographs and fluoroscopic images were obtained. As some rings are rigid and not deformable, the effect on the shape and hence the function of the SAPIEN XT

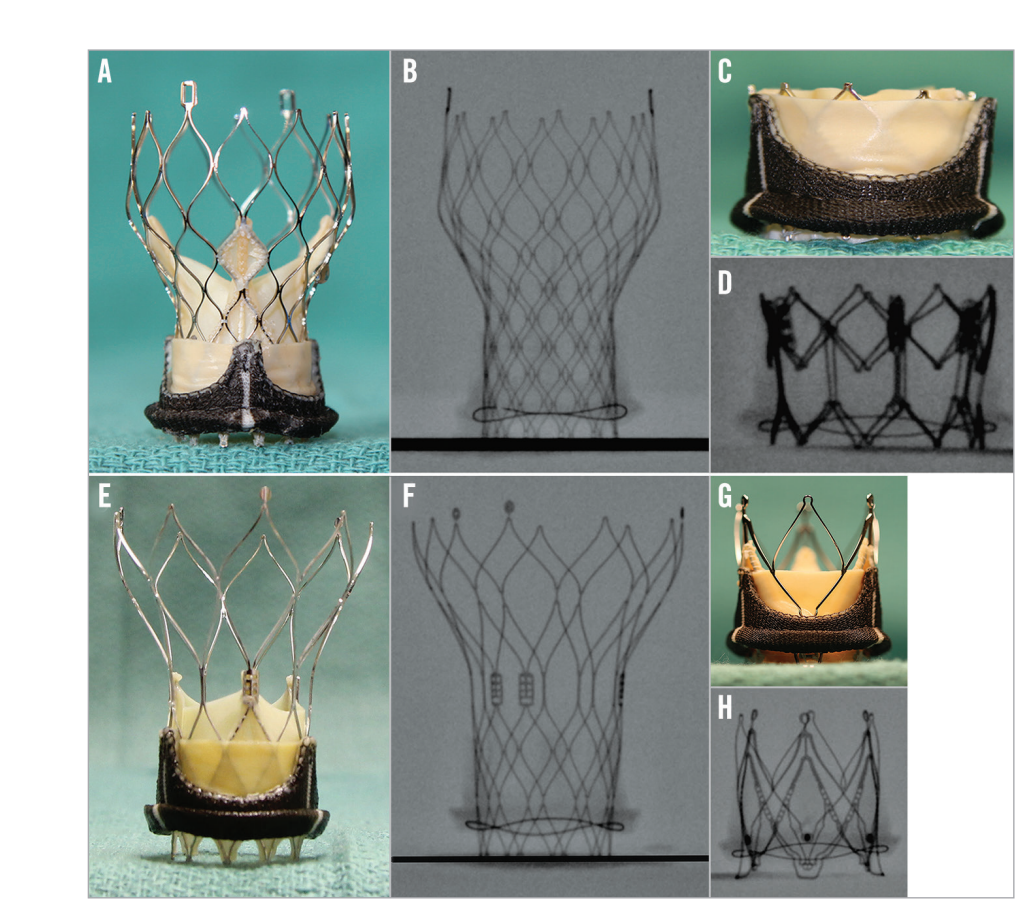

Figure 7. Ideal implant positions of commonly used THVs within an SHV. Example shown here is Soprano (Sorin). A) \& B) Ideal position of CoreValve, i.e., $4 \mathrm{~mm}$ below the sewing ring. C) \& D) Ideal position of SAPIEN XT, i.e., 10-15\% below the sewing ring. E) \& F) Ideal position of Portico, i.e., $4 \mathrm{~mm}$ below the sewing ring. G) \& H) Ideal position of JenaValve. 

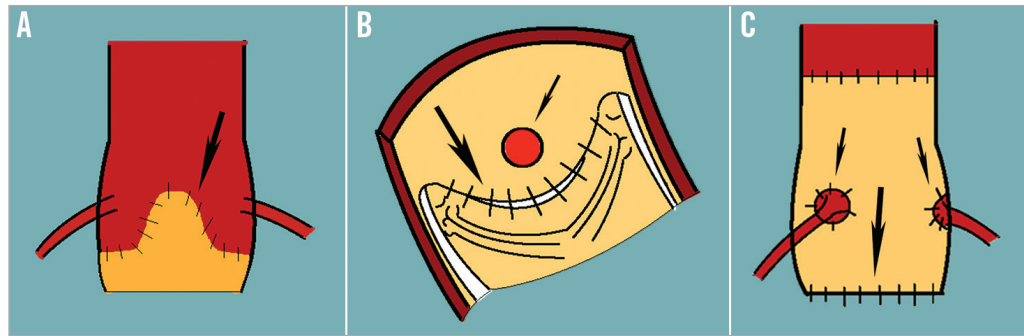

Figure 8. Schematic diagram demonstrating two methods of implanting stentless valves and their relative risk of coronary obstruction during a VIV procedure. Large arrow points to the suture line and small arrow to the coronary ostia. Subcoronary implantation of stentless valve - external appearance. Subcoronary implantation visualised from within the aorta, demonstrating the proximity of the suture line to the coronary ostia. Stentless valve implanted as a root replacement.

was documented to recommend the suitability of rings for a VIR procedure (Figure 9).

\section{TWO APPS: AORTIC AND MITRAL}

There are considerable differences between SHVs for aortic and mitral use. Rings are used only in the mitral position. Mitral VIV and VIR are associated with unique complications not seen with aortic VIV, i.e., delayed embolisation and left ventricular outflow tract obstruction. To address this, we have developed the VIV mitral app. Similar to the aortic app, the mitral app provides detailed information relevant to mitral VIV and VIR procedures. The workflow from the aortic app has been maintained, and it is easy for the users to familiarise themselves with the contents. An additional information section has useful links, including the differences to be considered between aortic and mitral VIV.

\section{Clinical relevance}

Some of the complications reported with the VIV procedure are coronary obstruction, malposition and residual gradients. The app provides warnings to draw the user's attention at relevant steps to help anticipate complications or a suboptimal result.

\section{SUBOPTIMAL FUNCTION}

If VIV is being considered, for example, in a $19 \mathrm{~mm}$ Perimount SHV (Edwards Lifesciences) whose true ID is $17 \mathrm{~mm}$, the app does not recommend the user to proceed with VIV using currently available THV sizes (Figure 10A). If selected, it connects to information on why it is not recommended. The user can then at least reconsider the decision to perform a VIV procedure, or proceed but with a suboptimal result. Similarly, when considering a VIR procedure in an IMR ETlogix mitral ring (Edwards Lifesciences), the user is warned of SAPIEN XT distortion, which may result in on-the-table regurgitation (Figure 10B).

\section{CORONARY OBSTRUCTION}

SHV models with pericardium sutured outside the stent frame (Mitroflow [Sorin, Milan, Italy] and Trifecta ${ }^{\mathrm{TM}}$ [St. Jude Medical]), when present in combination with shallow sinuses and a large oversize with a THV, can result in coronary obstruction ${ }^{11}$. The users are warned about this possibility and hence can take precautions by either placing coronary ostial wires or performing BAV to check the possibility of coronary obstruction before proceeding with VIV implantation (Figure 10C).

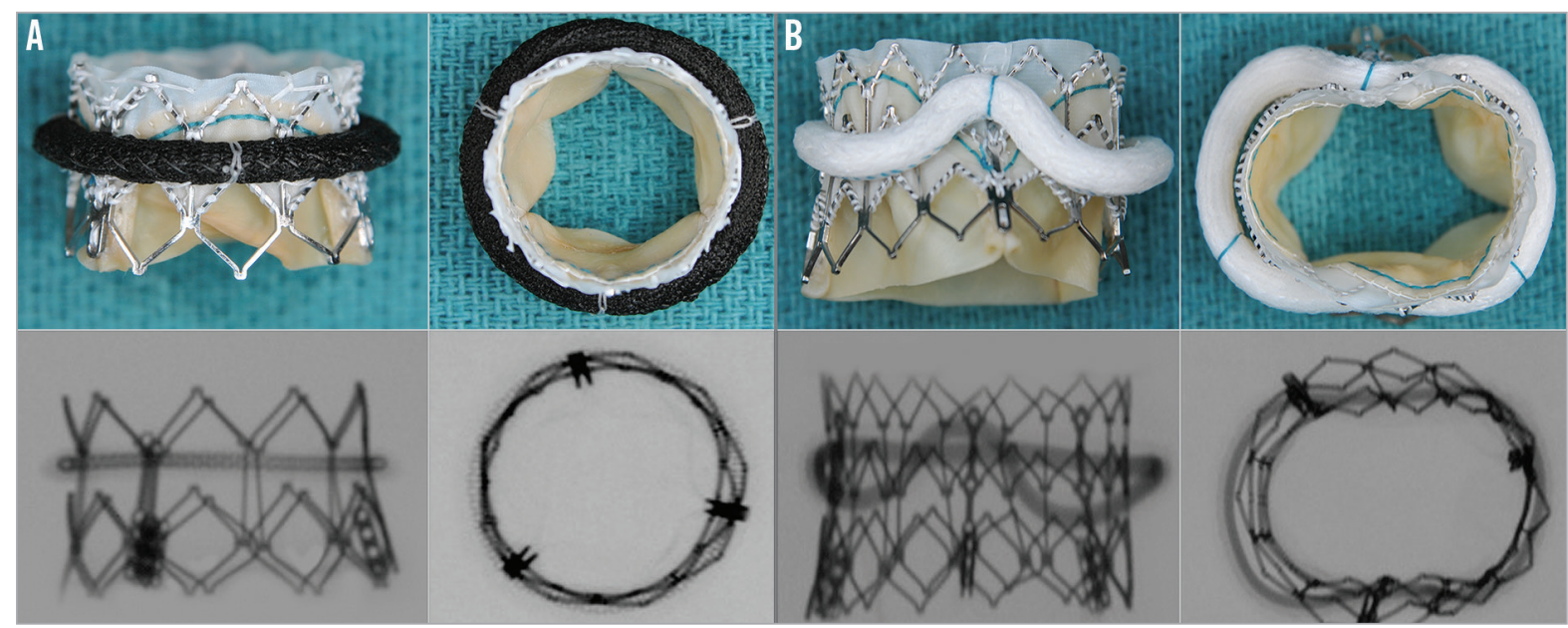

Figure 9. Compatibility of different rings with SAPIEN XT for VIR. A) Demonstrates example of a ring (Memo 3D; Sorin), which is suitable for VIR procedure. SAPIEN XT maintains a near circular shape. B) Demonstrates example of a ring (GeoForm; Edwards Lifesciences), which is unsuitable for VIR procedure as it deforms the SAPIEN XT valve. 


\section{MALPOSITION}

VIV in a stentless SHV and in Mosaic ${ }^{\circledR}$ (Medtronic) stented SHVs is fraught with a higher risk of malposition. The app provides users with movie clips to facilitate correct placement during a VIV in a stentless valve. Similarly, for the Mosaic SHV, the app provides clear guidance in the form of images and movies to place each type of THV in the optimal position (Figure 10D).

\section{ROLE OF MSCT}

MSCT characterisation of different SHVs and the correlation of what is seen on MSCT and the true ID is being undertaken by our group. For example, when the SHV is Trifecta, the ID measured on MSCT will closely correlate with the true ID as the stent is radiopaque and the leaflets are outside the stent frame, but, in Hancock ${ }^{\circledR}$ II (Medtronic), the MSCT ID may be at least 3 to $4 \mathrm{~mm}$ more than the true ID as the marker is in the sewing ring and it has porcine leaflets inside the stent. Apps give users most of the information required to perform a VIV when the SHV type and size are known. MSCT and 3D TEE have a role when the size is not known, but it is important to understand the design differences.

\section{How to use the app}

Search and download: the VIV aortic apps are free to download from the App Store and Google Marketplace. Once downloaded, they do not require an internet connection for access and use.

Workflow: the information is presented in such a way that the users can choose a specific SHV/ring relevant to the clinical scenario and familiarise themselves with important design information about that valve. They can then choose the appropriate label size to obtain important dimensions and also find out which size of THV valve type and sizes can be used. After selecting the THV valve type, the next link demonstrates how it is best placed during a VIV/ VIR procedure in the relevant SHV/ring. A short movie clip of an actual case can then be accessed when available.

Additional information: the app contains all relevant information on similar-looking valves/rings, fluoroscopic classification, true ID and unknown valve types, which will help the users to fine-tune the concept of VIV/VIR. In the fluoroscopic classification section, the concept of supra- and intra-annular models of SHV and its relevance to proper placement of THV is explored in detail.

Updates: two updates will be provided every year to coincide with the EuroPCR meeting and London PCR valve meeting as new THV devices are being approved and used increasingly for this indication.

\section{Conclusion}

VIV and VIR are exciting but complex new fields developing rapidly following the success of TAVI. To ensure a good clinical outcome, one must have a clear understanding of SHV/ring designs and their compatibility with THVs currently available. The apps provide this information at the user's fingertips with an easy-tonavigate platform providing patient-specific answers to the users. Information, such as a new THV device, can easily be added. Within one year of release, the apps have been downloaded in 90 countries with more than 10,000 downloads for the Aortic and 4,000 for the Mitral app, and this continues to rise with the popularity of VIV therapy in all corners of the world.

\section{Acknowledgements}

We would like to acknowledge Shalaka Bapat for providing the idea of the app, and Martyn Thomas, Martin Leon, Mark Monaghan, and Urmi Bapat for suggestions and feedback during the development stage of the app.

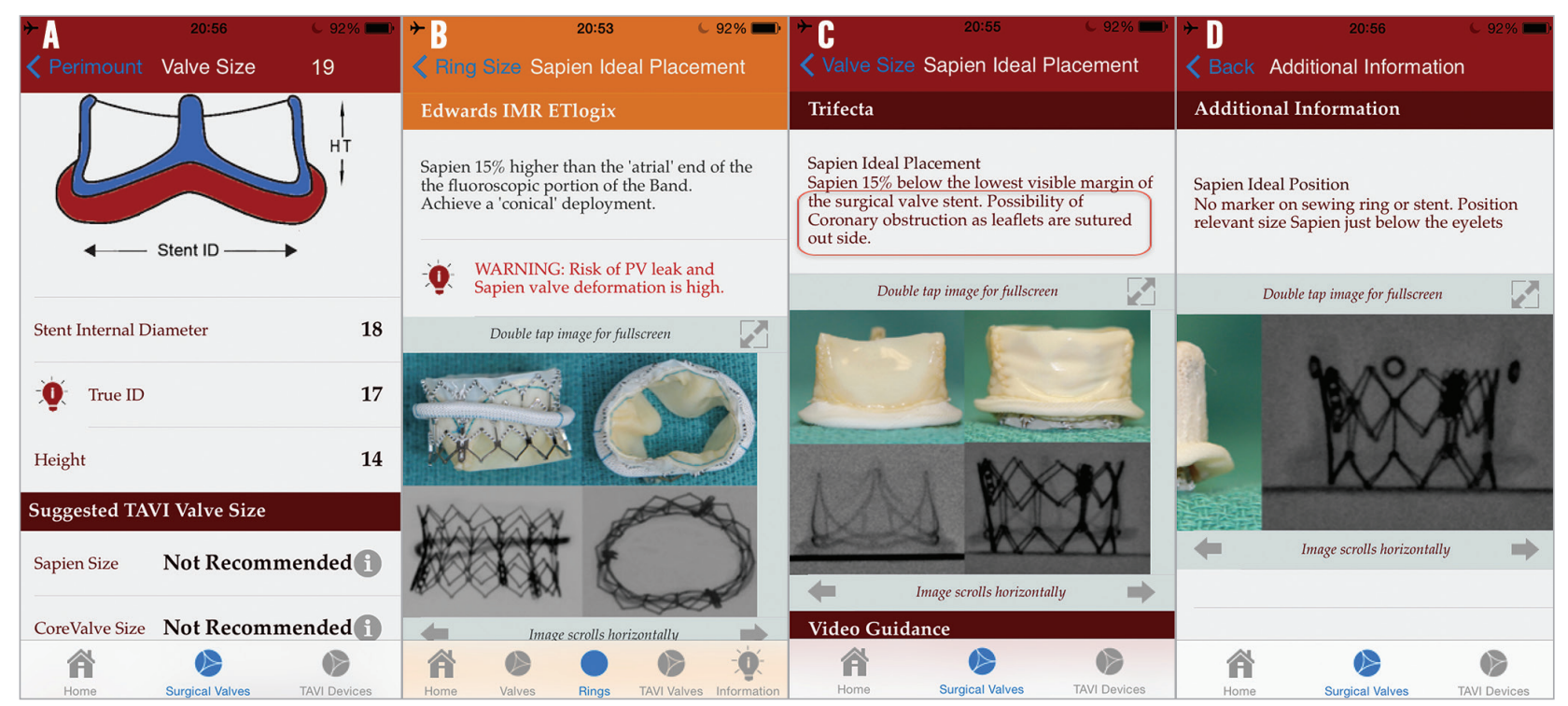

Figure 10. Importance of using the app to avoid complications. A) Screen after selecting 19 Perimount warns the user that the results after VIV will not be suitable with current sizes of THV. B) Screen warning the user about possibility of SAPIEN XT deformation and PV leak if VIR is performed within an IMR ring. C) Screen warning the user of a possibility of coronary obstruction after a VIV procedure within a Mitroflow (red box). D) Screen providing additional guidance for VIV procedure within a Mosaic SHV. 


\section{Conflict of interest statement}

V. Bapat is a consultant for Edwards Lifesciences, Medtronic Inc., and St. Jude Medical.

\section{References}

1. Cribier A. Development of transcatheter aortic valve implantation (TAVI): a 20-year odyssey. Arch Cardiovasc Dis. 2012;105:146-52.

2. Généreux P, Head SJ, Wood DA, Kodali SK, Williams MR, Paradis JM, Spaziano M, Kappetein AP, Webb JG, Cribier A, Leon MB. Transcatheter aortic valve implantation: 10-year anniversary part II: clinical implications. Eur Heart J. 2012;33:2399-402.

3. Bapat V, Attia R, Redwood S, Hancock J, Wilson K, Young C, Thomas M. Use of transcatheter heart valves for a valve-in-valve implantation in patients with degenerated aortic bioprosthesis: technical considerations and results. J Thorac Cardiovasc Surg. 2012;144:1372-9.

4. Gurvitch R, Cheung A, Ye J, WoodDA, Willson AB, Toggweiler S, Binder R, Webb JG. Transcatheter valve-in-valve implantation for failed surgical bioprosthetic valves. J Am Coll Cardiol. 2011;58:2196-209.

5. Khawaja MZ, Haworth P, Ghuran A, Lee L, de Belder A, Hutchinson N, Trivedi U, Laborde J, Hildick-Smith D. Transcatheter aortic valve implantation for stenosed and regurgitant aortic valve bioprostheses: CoreValve for failed bioprosthetic aortic valve replacements. J Am Coll Cardiol. 2010;55:97-101.

6. Latib A, Ielasi A, Montorfano M, Maisano F, Chieffo A, Cioni M, Mussardo M, Bertoldi L, Shannon J, Sacco F, Covello RD, Figini F, Godino C, Grimaldi A, Spagnolo P, Alfieri O, Colombo A. Transcatheter valve-in-valve implantation with the Edwards SAPIEN in patients with bioprosthetic heart valve failure: the Milan experience. EuroIntervention. 2012;7:1275-84.

7. Seiffert M, Diemert P, Koschyk D, Baldus S, Schirmer J, Meinertz T, Reichenspurner H, Treede H. Transapical implantation of a second generation transcatheter heart valve in patients with noncalcified aortic regurgitation. JACC Cardiovasc Interv. 2013;6: 590-7.

8. Fassa AA, Himbert D, Brochet E, Depoix JP, Cheong AP, Alkhoder S, Nataf P, Vahanian A. Transseptal transcatheter mitral valve implantation for severely calcified mitral stenosis. JACC Cardiovasc Interv. 2014;7:696-7.

9. Bapat V, Chadalavada S, Tehrani H, Attia R, Thomas M. A guide to fluoroscopic identification and design of bioprosthetic valves: a reference for valve-in-valve procedure. Catheter Cardiovasc Interv. 2013;81:853-61.

10. Bapat VN, Attia R, Thomas M. Effect of valve design on the stent internal diameter of a bioprosthetic valve: a concept of true internal diameter and its implications for the valve-in-valve procedure. JACC Cardiovasc Interv. 2014;7:115-27.

11. Piazza N, Bleiziffer S, Brockmann G, Hendrick R, Deutsch MA, Opitz A, Mazzitelli D, Tassani-Prell P, Schreiber C, Lange R. Transcatheter aortic valve implantation for failing surgical aortic bioprosthetic valve: from concept to clinical application and evaluation (part 1). JACC Cardiovasc Interv. 2011;4:721-32.

12. Cheung A, Webb JG, Barbanti M, Freeman M, Binder RK, Thompson C, Wood DA, Ye J. 5-year experience with transcatheter transapical mitral valve-in-valve implantation for bioprosthetic valve dysfunction. J Am Coll Cardiol. 2013;61:1759-66.

13. Descoutures F, Himbert D, Maisano F, Cassleman F, de Weger A, Bodea O, Van der Kley F, Colombo A, Giannini C, Rein KA, De Bruyne B, Petronio AS, Dahle G, Alfieri O, Vahanian A. Transcatheter valve-in-ring implantation after failure of surgical mitral repair. Eur J Cardiothorac Surg. 2013;44:e8-15.

14. Bothe W, Swanson JC, Ingels NB, Miller DC. How much septal-lateral mitral annular reduction do you get with new ischemic/functional mitral regurgitation annuloplasty rings? J Thorac Cardiovasc Surg. 2010;140: 117-21.

15. Shuto T, Kondo N, Dori Y, Koomalsingh KJ, Glatz AC, Rome JJ, Gorman JH 3rd, Gorman RC, Gillespie MJ. Percutaneous transvenous Melody valve-in-ring procedure for mitral valve replacement. J Am Coll Cardiol. 2011;58:2475-80.

16. Dvir D, Webb J, Brecker S, Bleiziffer S, Hildick-Smith D, ColomboA, DescouturesF, Hengstenberg C, MoatNE, BekeredjianR, Napodano M, Testa L, Lefevre T, Guetta V, Nissen H, Hernández JM, Roy D, Teles RC, Segev A, Dumonteil N, Fiorina C, Gotzmann M, Tchetche D, Abdel-Wahab M, De Marco F, Baumbach A, Laborde JC, Kornowski R. Transcatheter aortic valve replacement for degenerative bioprosthetic surgical valves: results from the global valve-invalve registry. Circulation. 2012;126:2335-44.

17. Conradi L, Kloth B, Seiffert M, Schirmer J, Koschyk D, Blankenberg S, Reichenspurner H, Diemert P, Treede H. The challenge of valve-in-valve procedures in degenerated Mitroflow bioprostheses and the advantage of using the JenaValve transctheter heart valve. EuroIntervention. 2014 Mar 20. pii: 20131122-01. [Epub ahead of print].

18. Jeger RV, Manoharan G, Kaiser CA. First-in-man Portico transcatheter aortic valve-in-valve implantation in a degenerated $19 \mathrm{~mm}$ Mitroflow aortic pericardial heart valve. EuroIntervention. 2014;9:1368.

19. Ihlberg L, Nissen H, Nielsen NE, Rück A, Busund R, Klaarborg KE, Soendergaard L, Harnek J, Miettinen H, Eskola M, Wahba A, Laine M. J Thorac Cardiovasc Surg. 2013;146:1047-54. 20. Piazza N, Bleiziffer S, Brockmann G, Hendrick R, Deutsch MA, Opitz A, Mazzitelli D, Tassani-Prell P, Schreiber C, Lange R. Transcatheter aortic valve implantation for failing surgical aortic bioprosthetic valve: from concept to clinical application and evaluation (part 2). JACC Cardiovasc Interv. 2011;4: 733-42.

21. Chevalier F, Leipsic J, Genereux P. Valve-in-valve implantation with a 23-mm balloon-expandable transcatheter heart valve for the treatment of a 19-mm stentless bioprosthesis severe aortic regurgitation using a strategy of "extreme" underfilling. Catheter Cardiovasc Interv. 2014;84:503-8.

22. Bapat VN, Attia RQ, Condemi F, Visagan R, Guthrie M, Sunni S, Thomas M. Fluoroscopic guide to an ideal implant position for Sapien XT and CoreValve during a valve-in-valve procedure. JACC Cardiovasc Interv. 2013;6:1186-94.

23. Noorani A, Bapat V. There's an App for That! Cardiac Interventions Today. 2014 July/ August, 42-6. 